\title{
Socio-Medical and Personality Correlates of Psychological Well-Being among People Living with HIV: a Latent Profile Analysis
}

\author{
Marcin Rzeszutek ${ }^{1}$ (D) Ewa Gruszczyńska ${ }^{2}$. \\ Ewa Firląg-Burkacka ${ }^{3}$
}

Received: 11 August 2017 / Accepted: 8 May 2018 /Published online: 5 June 2018

(C) The Author(s) 2018

\begin{abstract}
The aim of this study was to examine the heterogeneity of the sample of people living with HIV (PLWH) in terms of psychological well-being (PWB) using the latent profile analysis. Participants were 530 people with confirmed diagnosis of HIV+. The study was cross-sectional with psychological well-being operationalized by health related quality of life (WHO Quality of Life-BREF), satisfaction with life (Satisfaction with Life Scale) and positive and negative general affect (PANAS-X). The Big Five personality traits were measured with the NEO-FFI questionnaire. Additionally, sociomedical data were obtained. The statistical analysis proved the heterogeneity of PWB among the studied patient group: model with 6 different PWB profiles was best-fitted to the data. Employment, education, and personality traits, especially neuroticism, turned out to be significant for PWB profile membership. In contrast, medical variables were not related to the PWB profile membership among participants. Research on PWB among PLWH in so called person-centred approach by distinguishing their different subgroups may be an adjunct to develop more effective methods of psychological counselling for PLWH, especially in the light of findings that objective health status is not necessarily a strong explanatory variable of PWB's variance in these patients.
\end{abstract}

Marcin Rzeszutek

marcin.rzeszutek@psych.uw.edu.pl

Ewa Gruszczyńska

egruszczynska@swps.edu.pl

Ewa Firlag-Burkacka

burkacka@ poczta.onet.pl

1 Faculty of Psychology, University of Warsaw, Stawki 5/7, 00-183 Warsaw, Poland

2 Faculty of Psychology, University of Social Sciences and Humanities, Chodakowska 19/31, 03-815 Warsaw, Poland

3 Warsaw's Hospital of Infectious Diseases, Wolska 37, 01-201 Warsaw, Poland 
Keywords HIV· Psychological well-being $\cdot$ Personality $\cdot$ Latent profile analysis

Over the past few decades researchers and practitioners have been emphasizing the centrality of helping chronically ill patients achieve optimal functioning and well-being, i.e. improving the quality of life (QoL) (Ware and Sherbourne 1992). Several authors have also discussed the connection between health and QoL, and argued for differentiating between these concepts (Bullinger 2002). Therefore, the term health related quality of life (HRQoL) was introduced to solve the problem that QoL denotes a variety of medical as well as non-medical categories (Guyatt et al. 1993). Nevertheless, the HRQoL construct has been also criticized for being mainly pathogenetically oriented and concentrated on disability caused by a particular disease, hardly ever considering the possibility of satisfactory life in spite of an illness (Constanza et al. 2007). With the emergence of positive psychology, increasing number of authors started to look beyond HRQoL by focusing on improving subjective well-being (SWB) (Alayli et al. 2014). SWB is usually defined as people's satisfaction with their lives as a whole or with the individual domains of their lives and includes two main components: satisfaction with life, which is the cognitive aspect of subjective well-being and affective experiences and moods, i.e., positive and negative emotional reactions to peoples' life (Diener et al. 2010). It was proved that high levels of SWB are related to good health, longevity, and optimal human functioning (Steptoe et al. 2015). However, till now studies on psychological well-being among chronically ill patients have been mainly concentrated on negative aspects of well-being, i.e. they were usually focused merely on the absence of mental disorders, such as depression or anxiety (Logie and Gadalla 2009; Moskowitz 2003). Taking into an account this shortcoming, we have concentrated in this study on psychological well-being (PWB) operationalized broadly by the level of health related quality of life (HRQoL; The WHOQOL Group 1995), the level of satisfaction with life (SWL) and a combination of positive and negative affect (PA/NA; Watson et al. 1988; Fredrickson 2013) among people living with HIV (PLWH).

The substantial progress in antiretroviral therapy has led increasing number of authors to claim that HIV infection is perceived more a chronic than as terminal illness (Deeks et al. 2013). Moreover, the recent advance in knowledge about the disease and new treatments has provided great hope for PLWH to live a longer life (Samji et al. 2013). Nevertheless, PLWH still suffer from intense psychological distress resulting from being diagnosed with a potentially life-threatening virus (Rzeszutek and Oniszczenko 2013, Rzeszutek et al. 2015), unpredictability of HIV progression (Jayarajan and Prabha 2010) and social stigmatization (Bogart et al. 2011; Chi et al. 2016). HIV-related distress often causes mental health problems among PLWH, which may not only deteriorate PWB, but also low PWB can influence the course of HIV infection by lowering CD4 cell counts and increasing the level of physical HIV symptoms (Pacella et al. 2012). For these reasons research on PWB among people living with HIV becomes a great concern (Darling et al. 2010; Tsevat et al. 2009). Nevertheless, studies on PWB among PLWH have mainly been focused on HRQoL, which covers only limited PWB areas of this population and there is a need to incorporate also subjective well-being (SWB) of this patient group (Oberjé et al. 2015). 
Some authors showed that PWB is a subjective phenomenon shaped greatly by personal attributes, of which personality traits play a vital role (Erlen et al. 2009; Whitworth et al. 2013). As far as PLWH is concerned, it has been proved that personality traits may be correlates of HIV-related mental health mental health problems (see, posttraumatic stress symptoms, Rzeszutek and Oniszczenko 2013) and affect patients' quality of life (Erlen et al. 2009). In particular, it was found that personality traits postulated by the Big Five taxonomy may be linked to medication adherence (see, neuroticism; Penedo et al. 2003; extraversion, Kumar 2015), are related to slower decrease of CD4 count (see, conscientiousness; O'Cleirigh et al. 2007) and are associated with lower perceived stigmatization (see, openness to experience; McCrae et al. 2007). However, previous studies on aforementioned topic precluded the possibility of obtaining a more comprehensive insight into psychological well-being among this patient group, i.e., to capture the heterogeneity of psychological well-being in relation to personality traits.

The impact of some sociodemographic factors such as socioeconomic status and marital status and on PWB is relatively consequent, i.e. low socioeconomic status, single/nonmarital status are all related to poorer PWB among PLWH (Asakawa et al. 2011). Conversely, research on the role of other sociodemographic data and PWB among this patient group is inconclusive and needs further investigation (Gordillo et al. 2009). For example, regarding sex, it is recognized that HIV+ women usually receive lower scores on particular well-being dimension (Cherepanov et al. 2011). On the other hand, some studies found that HIV+ women were characterised by overall better physical health due to use of more adaptive coping strategies compared to HIV+ men (Liu et al. 2006). Finally, sociodemographic factors, particularly age and sex, may have different impact on clinical and disease-related factors among PLWH, and the mechanism, which underlies these differences, is not entirely known (Degroote et al. 2014). It is likely that these conflicting results may be caused by the fact that many authors neglected the problem of heterogeneity of PLWH in respect to socio-medical variables and its various impacts on different PWB dimensions.

\section{Current Study}

The aim of this study was to examine the heterogeneity of the studied sample of PLWH in respect to PWB using the latent class analysis (see, data analysis). In particular, we wanted to identify correlates of PWB profiles' membership among socio-medical data, as well as personality dimensions. To our knowledge there are no studies conducted among PLWH, which could serve us as a direct source of relevant hypothesises in case of this particular study design. Therefore, mainly an exploratory approach has been employed in order to identify the number of PWB latent classes in our sample. However, basing on aforementioned studies on PWB and its determinants among PLWH, we expected that socio-medical data will differ between groups with better and worse PWB profiles. Additionally, neuroticism, conscientiousness, openness to experience and extraversion were supposed to be a significant correlates of PWB profiles, i.e. lower level of neuroticism, higher level of: conscientiousness, openness to experience and extraversion will be associated with better PWB profiles. 


\section{Method}

\section{Participants and Procedure}

The sample was comprised of 530 adults with a medical diagnosis of HIV infection. In particular, from the 750 patients eligible for the study, 530 were recruited and agreed to filled out questionnaires (71\%), 152 declined (20\%), and $68(9 \%)$ completed the questionnaires with a level of missing data, which precluded including them into the statistical analysis. Patients were recruited from the state hospital of infectious diseases out-patient clinic. The questionnaires were distributed in paper form by the first author of this study and professional interviewers. After the informed consent the participants filled out a paper-and-pencil version of the questionnaires and participated in the study voluntarily, as there was no remuneration for the participation. The eligibility criteria were 18 years of age or older, confirmed medically diagnosis of HIV+ and receiving care from the hospital where the study was conducted. The exclusion criteria encompassed HIV-related cognitive disorders, which were screened by medical doctors. The study was approved by the local ethics commission. Table 1 summarizes socio-medical variables, which characterised our sample.

Table 1 Socio-medical variables in the studied sample $(N=530)$

\begin{tabular}{|c|c|}
\hline Variable & $\mathrm{N}(\%)$ \\
\hline \multicolumn{2}{|l|}{ Gender } \\
\hline Male & $444(83.8 \%)$ \\
\hline Female & $86(16.2 \%)$ \\
\hline Age in years $(\mathrm{M} \pm \mathrm{SD})$ & $39.81 \pm 10.54$ \\
\hline \multicolumn{2}{|l|}{ Marital status } \\
\hline Married & $333(62.8 \%)$ \\
\hline Single & $227(37.2 \%)$ \\
\hline \multicolumn{2}{|l|}{ Education } \\
\hline Elementary & $84(15.8 \%)$ \\
\hline Secondary & $162(30.6 \%)$ \\
\hline University degree & $284(53.6 \%)$ \\
\hline \multicolumn{2}{|l|}{ Employment } \\
\hline Full employment & $384(72.5 \%)$ \\
\hline Unemployment & $61(11.5 \%)$ \\
\hline Retirement & $21(3.9 \%)$ \\
\hline Sickness Allowance & $64(12.1 \%)$ \\
\hline \multicolumn{2}{|l|}{ HIV/AIDS status } \\
\hline HIV+ only & $448(84.5 \%)$ \\
\hline HIV/AIDS & $82(15.5 \%)$ \\
\hline HIV infection duration in years $(\mathrm{M} \pm \mathrm{SD})$ & $7.77 \pm 6.86$ \\
\hline Antiretroviral treatment $(\mathrm{ART})$ duration in years $(\mathrm{M} \pm \mathrm{SD})$ & $5.97 \pm 5.53$ \\
\hline CD4 Count & $589.46 \pm 222.42$ \\
\hline
\end{tabular}

M, mean; SD, standard deviation 


\section{Measures}

\section{Well-Being Indicators}

Health related quality of life (HRQoL) was measured with the WHO Quality of LifeBREF (WHOQOL-BREF), developed by a group of under the WHO initiative to assess this construct cross-culturally (WHOQOL Group 1995). WHOQOL-BREF comprises of 26 items to measure four domains: somatic health, psychological health, social relationships, and environment. The higher values indicate higher quality of life in each domain. Cronbach's alpha coefficients for the current study were satisfactory and exceeded .86 for aforementioned HRQoL domains.

Subjective well-being was assessed with the Satisfaction with Life Scale (SWLS; Diener et al. 1985), together with the Positive and Negative Affect (PANAS-X; Watson et al. 1988). SWLS, consists of five items, where respondents evaluate each item on a 7-point scale ranging from 1 (strongly disagree) to 7 (strongly agree). Therefore, a higher total score on this scale indicates higher level of satisfaction with life. Cronbach's alpha coefficient in the studied sample was .88 .

Positive and negative affect was assessed with 20 descriptions of feelings and emotions: 10 for positive affect (e.g., 'proud', 'excited') and 10 for negative affect (e.g., 'depressed', 'stressed') from the PANAS-X by Watson et al. (1988). In this inventory participants rate their general affective states on a 5-point response scale from 1 - not at all to 5 - strongly. Cronbach's alpha coefficients obtained in this study for positive affect scale was .85 and for negative affect scale .86 .

\section{Personality Dimensions}

Personality traits were evaluated with the NEO-FFI questionnaire by Costa Jr. and McCrae (1992). NEO-FFI consists of 60 items (12 per each trait), to which participants respond on a 5-point scale from strongly disagree to strongly agree. Five indices were obtained: neuroticism, extraversion, openness to experience, agreeableness and conscientiousness. The higher scores of each informs about higher level of each trait. The Cronbach's alpha for the current study ranged from .72 to .84 .

\section{Data Analysis}

Latent class analysis (LCA) allows to examine unobserved heterogeneity within a studied sample, i.e., it enables to identify groups of participants, who represent the greatest similarity on the same set of observed variables within a given group and the greatest dissimilarity between other participants' groups (Lubke and Neale 2006). The latent variable in LCA is categorical with a number of categories equal to a number of classes (Vermunt and Magidson 2004). The most popular information-theoretic methods are Akaike's Information Criterion (AIC) and Bayesian Information Criterion (BIC) as well as the sample-size adjusted BIC (SABIC), where the lowest values in a given set of competing models indicate a model with the best fit (Vermunt 2010). Finally, another method is the bootstrap likelihood ratio test (BLRT), which compares neighboring models with $\mathrm{k}-1$ and $\mathrm{k}$ classes, respectively. Entropy informs about 
classification error and values closer to 1 indicates better class separation (Collins and Lanza 2013). Finally, a size of the smallest class is a criterion of practical importance since classes smaller than $5 \%$ of the sample is considered hard to replicate.

When continuous observed indicators are used in LCA, it has been referred to as a latent profile analysis (LPA, Collins and Lanza 2013), which was the case in this study. Since we did not want covariates to influence the determination of class membership, we used the so called three-step approach. First, classes were identified on the basis of six observable, i.e., continuous indicators describing different domains of PWB among PLWH. In the second step, the participants were assigned to latent classes on the basis of their probability scores, and, in the third and last step, these probability scores were related to socio-medical data as well as to personality dimensions. Such an approach allows us to avoid bias caused by treatment of categorical latent variable (class membership) as an observable one and is superior to commonly used techniques of between-class mean analysis or logistic regression with modal allocation to classes as a dependent variable. Specifically, we used the Bias-Adjusted Step-Three Analysis with the ML (maximum likelihood) adjustment method proposed by Vermunt (2010), recommended for model with covariates. The calculations were performed using the Latent GOLD 5.1 (submodule called Step3 included) and IBM SPSS Statistics version 24.

\section{Results}

\section{Descriptive Statistics}

Table 2 presents descriptive statistics and Person's correlations for PWB and personality dimensions among our participants. As can be seen, for all the variables absolute values of both skewness and kurtosis are below 1, which is considered satisfactory to assume a normal distribution (Gravetter and Wallnau 2014). All PWB indicators correlated within .3-.7 range. The only exception was an independence of positive and negative affect $(r=-.02)$. Relationships between PWB and personality dimensions were more complex and the highest number of significant correlations has been found for neuroticism and extraversion. Importantly, no extreme correlations were noted thus there was no variable overlapping.

\section{Profiles of Psychological Well-Being}

Neighboring models from 1 up to 8 classes were tested. Model fitting was stopped when the size of the smallest class appeared to be less than $5 \%$ of the total sample. The fit statistics are presented in Table 3. As can be seen, there is no coherence in values of information-theoretic indices: the lowest BIC and SABIC suggested a 6-class solution, whereas AIC pointed out at a 8-class solution. The BLRT informs that each successive model above a one-class model provided a significant improvement. When entropy and class sizes have been taken into account, models with more than 6 classes are regarded as being fitted worse to the data. Thus, model with 6 classes has been chosen for further inspection. The class profiles are plotted in Fig. 1 using Z-scores given the differences in 


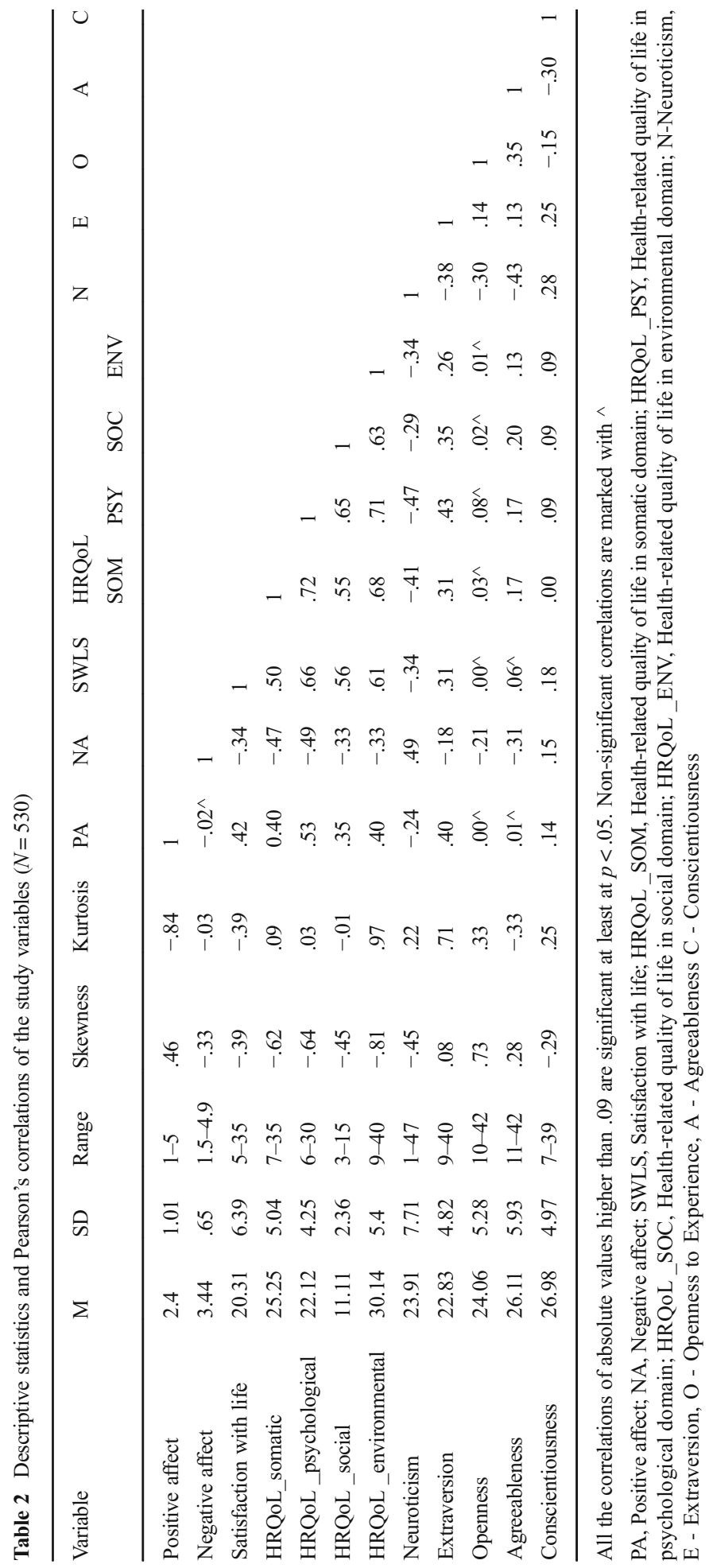


Table 3 Summary of model selection indices of latent prolife analysis

\begin{tabular}{|c|c|c|c|c|c|c|c|}
\hline Model & $\mathrm{BIC}$ & AIC & SABIC & Number of Entropy & BLRT & & Smallest class \\
\hline & & & & parameters & value & $p$ & $\%$ of $\mathrm{N}$ frequency \\
\hline
\end{tabular}

\begin{tabular}{lrrrrrrrrr}
\hline 1-Class & $18,083.77$ & $18,023.95$ & $18,039.33$ & 14 & & & & & \\
2-Class & $16,797.52$ & $16,673.61$ & $16,705.47$ & 29 & 0.88 & 1380.34 & $<.001$ & $36.41 \%$ & 193 \\
3-Class & $16,512.55$ & $16,324.54$ & $16,372.88$ & 44 & 0.85 & 379.07 & $<.001$ & $25.85 \%$ & 137 \\
4-Class & $16,378.95$ & $16,126.85$ & $16,191.67$ & 59 & 0.86 & 227.69 & $<.001$ & $9.06 \%$ & 48 \\
5-Class & $16,301.94$ & $15,985.75$ & $16,067.04$ & 74 & 0.86 & 171.11 & $<.001$ & $9.06 \%$ & 48 \\
6-Class & $16,299.34$ & $15,919.06$ & $16,016.83$ & 89 & 0.86 & 96.68 & $<.001$ & $7.92 \%$ & 42 \\
7-Class & $16,350.41$ & $15,906.03$ & $16,020.28$ & 104 & 0.84 & 43.03 & 0.022 & $6.04 \%$ & 32 \\
8-Class & $16,396.66$ & $15,889.19$ & $16,019.92$ & 119 & 0.82 & 46.84 & 0.006 & $5.09 \%$ & 27 \\
\hline
\end{tabular}

BIC, Bayesian Information Criterion; AIC, Akaike's Information Criterion; SABIC, Sample-Size Adjusted BIC; BLRT, Bootstrap Likelihood Ratio Test

scales of variables measurement. In order to facilitate an interpretation, the scores for negative affect were recorded in a way that higher values indicate higher PWB.

The profiles were quite coherent with only exception in respect to a profile for class 4 , which had a different pattern. Therefore, class $1\left(\mathrm{n}_{1}=145,27 \%\right)$ could be described as medium psychological well-being; class $2\left(\mathrm{n}_{2}=138,26 \%\right)$ as low psychological wellbeing, class $3\left(\mathrm{n}_{3}=114,22 \%\right)$ as high psychological well-being; class $4\left(\mathrm{n}_{4}=56,11 \%\right)$ as mixed psychological well-being; class $5\left(\mathrm{n}_{5}=44,8 \%\right)$ as very low psychological wellbeing and class $6\left(\mathrm{n}_{6}=32,6 \%\right)$ as very high psychological well-being. For comparison purposes, the medium psychological well-being profile could be considered as a referent profile since it is the most numerous and its values for satisfaction with life and quality of life are close to the sample averages see, horizontal line at Fig. 1.

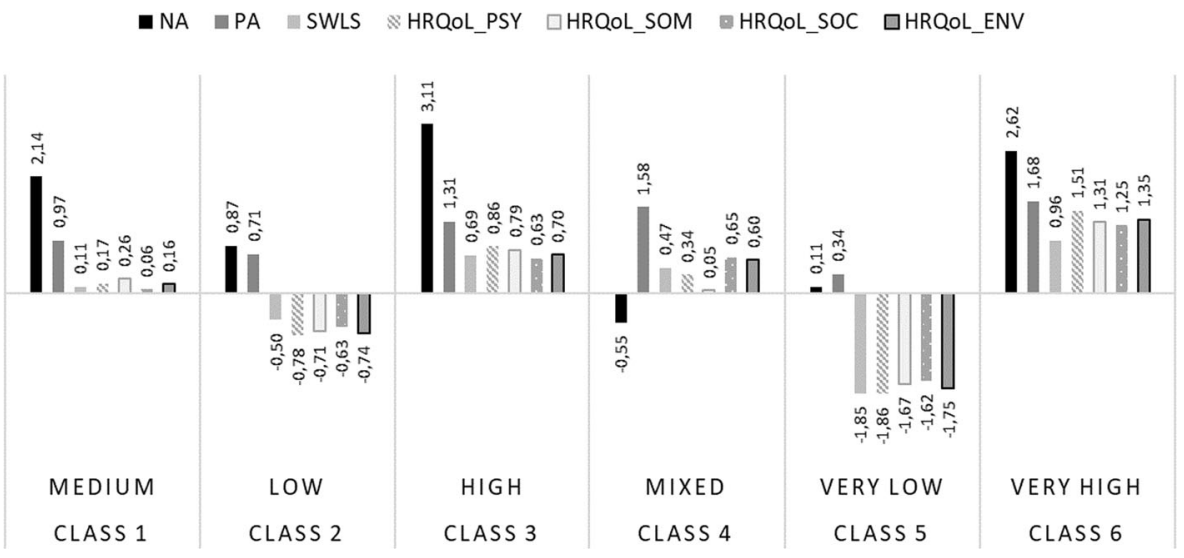

Fig. 1 Psychological well-being profiles in the studied sample $(N=530)$. Note. The higher values $(\mathrm{z}$-scores, above bars) of each variable indicate higher psychological well-being. NA - Negative affect; PA- Positive affect; SWLS - Satisfaction with life; HRQoL_PSY - Health-related quality of life in psychological domain; HRQoL_SOM - Health-related quality of life in somatic domain; HRQoL_SOC - Health-related quality of life in social domain; HRQoL_ENV - Health-related quality of life in environmental domain 


\section{Differences within PWB Profiles: Socio-Medical Variables and Personality Traits as Covariates}

In the last step of the 3-step approach adopted in this study, significant covariates of profile's membership were identified. Particularly, for each participant probabilities of belonging to each of 6 classes were saved as posteriors and then were related to potential covariates with ML bias-adjustment method. Model with class independent error variances was assumed. Nominal socio-medical variables were recoded into zero-one values. The results are presented in Table 4.

Importantly, medical variables did not predict class membership, so a proxy of objective physical health was irrelevant to well-being profiles. Among sociomedical data only education and current employment status appeared to be significant correlates of class membership. Figure 2 depicts both effects using distribution of proportion of participants with university degree to participants with lower level of education and proportion of employed participants to unemployed ones within classes.

Finally, for personality dimensions, all traits, except for agreeableness, revealed to be significant correlates of class membership. As can be seen on Fig. 3, for the farthest ("extreme") PWB profiles a clear differential pattern was observed. Namely, higher neuroticism, lower extraversion, lower openness to experience and lower conscientiousness were correlates of belonging to the very low PWB profile, whereas the reverse values of these dimensions were correlates of belonging to the very high PWB profile. Mixed PWB profile did not differ from medium PWB profile in terms of personality traits, which was also the case for the pair of high and very high PWB profile. In general, neuroticism yielded 12 out of 15 possible significant differences between profiles, extraversion -8 , consciousness 6 and openness to experience -5 .

\section{Discussion}

The results of our study revealed the heterogeneity of the sample of people living with HIV in respect to psychological well-being as 6 different profiles were obtained. This result is important itself, especially in the light of the contemporary studies on aforementioned topic, which were mainly concerned with identifying single sociomedical and/or psychological variables that are independently associated with various aspects of PWB in this patient group (Degroote et al. 2014). Keiser et al. (2012) in one of the few studies using latent profile analysis among PLWH suggested that a possible reason for many conflicting results in the literature on the PWB among PLWH is the fact that the majority of authors neglected how particular socio-medical and psychosocial variables may cluster across different HIV+ patients groups distinguished on the basis of various levels of PWB, which may be influenced by a variety of factors simultaneously. This study addressed the gap in the literature by examining sociomedical and personality covariates after identifying groups of PLWH that are similar with regard to different domains of PWB.

Firstly, the results of our study showed that objective physical health measured by selected medical variables was irrelevant to well-being profiles among PLWH. This 
Table 4 Results of the bias-adjusted stept-three analysis of correlates of the well-being profile membership

\begin{tabular}{|c|c|c|c|c|c|c|c|c|c|c|c|c|c|c|c|c|c|c|}
\hline \multirow{3}{*}{ Covariates } & \multirow{3}{*}{ Wald } & \multirow{3}{*}{$p$} & \multicolumn{15}{|c|}{ Paired comparisons between well-being profiles } & \multirow{3}{*}{$\begin{array}{l}\text { Number of } \\
\text { significant } \\
\text { effects }\end{array}$} \\
\hline & & & 1 & 1 & 1 & 1 & 1 & 2 & 2 & 2 & 2 & 3 & 3 & 3 & 4 & 4 & 5 & \\
\hline & & & 2 & 3 & 4 & 5 & 6 & 3 & 4 & 5 & 6 & 4 & 5 & 6 & 5 & 6 & 6 & \\
\hline \multicolumn{19}{|l|}{ Sociodemographic } \\
\hline Sex & 3.89 & .57 & & & & & & & & & & & & & & & & \\
\hline Relationship status & 4.98 & .42 & & & & & & & & & & & & & & & & \\
\hline Education & 10.89 & .05 & & & & 9.80 & & & & 5.52 & & & 8.60 & & 4.14 & & 5.20 & 5 \\
\hline Employment & 19.03 & .002 & 7.74 & & & & 4.12 & 8.49 & & & 8.52 & & 4.12 & & & 4.13 & 6.36 & 7 \\
\hline \multicolumn{19}{|l|}{ Medical } \\
\hline CD4 & 6.39 & .27 & & & & & & & & & & & & & & & & \\
\hline $\begin{array}{l}\text { Years since HIV } \\
\text { diagnosis }\end{array}$ & 2.14 & .83 & & & & & & & & & & & & & & & & \\
\hline $\begin{array}{l}\text { Years of ARV } \\
\text { treatment }\end{array}$ & 3.23 & .66 & & & & & & & & & & & & & & & & \\
\hline AIDS diagnosis & 8.19 & .15 & & & & & & & & & & & & & & & & \\
\hline \multicolumn{19}{|l|}{ Personality traits } \\
\hline Neuroticism & 57.88 & $<.001$ & 15.31 & 14.43 & & 24.73 & 11.41 & 41.34 & & 7.61 & 25.73 & 8.32 & 41.87 & & 5.48 & 8.30 & 34.97 & 12 \\
\hline Extraversion & 17.58 & .004 & 3.51 & & & & 3.82 & 7.59 & 9.05 & & 7.22 & & 5.55 & & 8.00 & & 7.56 & 8 \\
\hline $\begin{array}{l}\text { Openness to } \\
\text { experience }\end{array}$ & 12.578 & .028 & & & & 4.06 & & & & 6.70 & & & 7.37 & & 8.22 & & 3.87 & 5 \\
\hline Agreeableness & 4.05 & .54 & & & & & & & & & & & & & & & & \\
\hline Conscientiousness & 15.49 & .009 & & & & 9.93 & & & & 5.96 & 3.78 & & 7.50 & & 11.42 & & 10.00 & 6 \\
\hline
\end{tabular}

Note. For paired comparisons between classes of well-being profiles only Wald's values significant at least at $p<.05$ are presented

In the field headings: 1 - medium psychological well-being $\left(\mathrm{n}_{1}=145\right) ; 2$ - low psychological well-being $\left(\mathrm{n}_{2}=138\right) ; 3$ - high psychological wellbeing $\left(\mathrm{n}_{3}=114\right) ; 4$ - mixed psychological well-being $\left(\mathrm{n}_{4}=56\right) ; 5$ - very low psychological well-being $\left(\mathrm{n}_{5}=44\right) ; 6$ - very high psychological wellbeing $\left(\mathrm{n}_{6}=32\right)$.

result was intriguing, as several previous studies indicated that PWB among PLWH is associated with HIV infection duration (Rzeszutek et al. 2015), and CD4 count (Armon and Lichtenstein 2012). In our sample, however, aforementioned medical variables did not predict class membership, which may have important clinical implications. This result corresponds with other studies, which proved that well-being among people suffering from various chronic diseases depends in some cases more on psychosocial factors rather than health status and clinical variables (Simmons et al. 2000). Nevertheless, the result obtained in our sample can be interpreted also from another perspective. Some findings revealed that poor affective well-being and depression in particular, may be a more important predictor of PWB among PLWH than clinical variables or neuropsychological disorders (Trépanier et al. 2005). In addition, Vera-Villarroel et al. (2016) observed that both affect valences (positive and negative) may impact the quality of life among PLWH, but only negative affect was a mediator between this outcome and personality dimensions. It is worth mentioning that in our sample, on average, higher negative than positive affect was noted, which may be a sign of a relatively low level of affective well-being. Additionally, well-being profiles were the most diverse within negative affect domain. Thus, it suggests that affectivity is indeed a separate dimension of PWB and relation of positive to negative affect can differ significantly within the sample of people suffering from the same chronic illness.

Secondly, among the socio-medical data only employment and education were significantly related to the level of PWB, i.e. having a university degree and being employed were correlates of membership to higher PWB profile. In the context of insignificant effects of medical variables perhaps an employment can serve as a better proxy of patients' health. Being employed provides this kind of social participation which may be of special value for chronically ill patients (Vassilev et al. 2014). For PLWH this effect may be even stronger due to a threat of social rejection, including losing social status, job and financial resources (Samson et al. 2009). On the one hand, several studies highlighted the 
PROPORTION

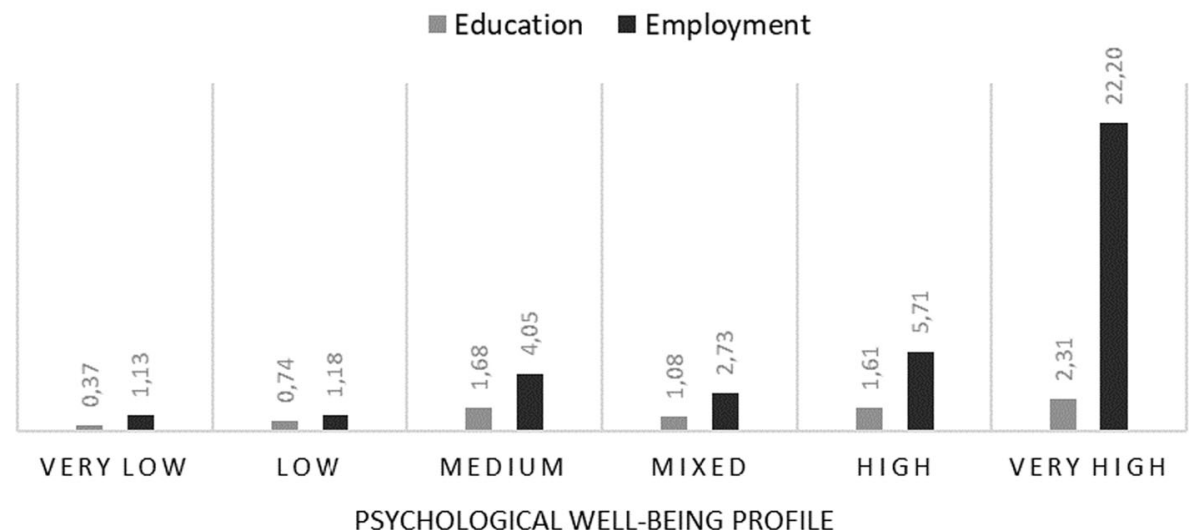

Fig. 2 Education and employment as psychological well-being profile covariates: proportion of participants with higher education and full employment for each profile. Note. Proportion for education: number of participants with university degree/number of participants with lower level of education. Proportion for employment: number of participants with full employment/number of participants without employment

importance of employment and education in maintenance a good health-related quality of life and psychological adjustment among PLWH (e.g. Degroote et al. 2014; Odek 2013; Logie and Gadalla 2009). On the other hand, this result should be discussed in the light of the recent advance in knowledge about the disease, new treatments and great progress in antiretroviral therapy, so increasing number of HIV+ individuals are not so preoccupied with their HIV infection and changed their attitude towards HIV/AIDS from a fatal and terminal illness to a chronic medical condition (Deeks et al. 2013).

Thirdly, all personality traits, except for agreeableness, revealed to be significant correlates of PWB among the studied patient group. It corresponds with other studies, which proved that these NEO traits, i.e., neuroticism, extraversion, openness to experience and conscientiousness are significantly associated with several aspects of PWB

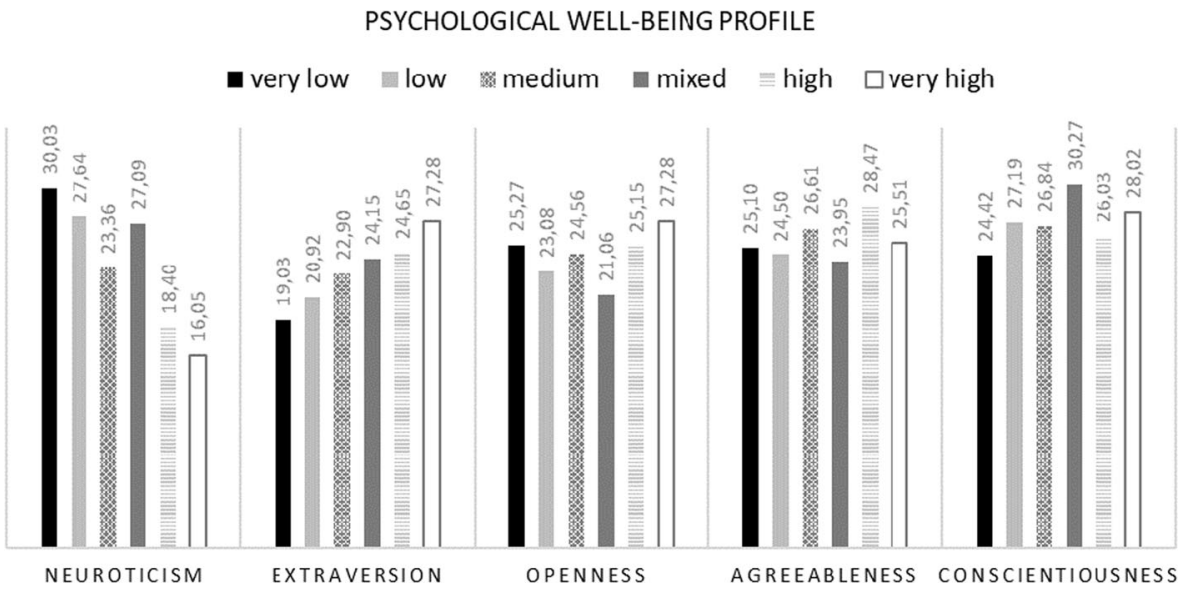

Fig. 3 Personality dimensions as correlates of psychological well-being profiles. Note. Relevant mean values above bars 
among PLWH (Fassino et al. 2004; Kumar 2015; McCrae et al. 2007). Interestingly, above mentioned effect was the most pronounced for neuroticism, i.e., neuroticism occurred to be the most salient correlate of class membership among our participants compared to all other examined variables. This result may be interpreted from a broader perspective of contemporary studies on personality, which showed an increasing significance of personality dimensions on various aspects of health and well-being, often outweighing the role of other psychological variables, as well as socio-medical data factors (Friedman and Kern 2013). As far as PLWH is concerned, neuroticism was particularly related to various aspects of PWB. For example, Burgess et al. (2000) observed a strong impact of neuroticism on HRQoL, independent of medical (CD4 count, HIV progression). Furthermore, Lockenhoff et al. (2009) found neuroticism as the strongest predictor of low mental health among PLWH, even after controlling for socio-medical data.

\section{Limitations}

It is important to mention several limitations of this study. First of all, the crosssectional design of this study precludes causal interpretations of the relationships between variables observed among our participants. Secondly, as already mentioned, prior studies on this topic have focused on HRQoL, and the novelty of the current study comes from combining it with assessment of SWB, i.e., satisfaction with life and affective well-being. However, on the basis of the results, it can be stated that concentration on SWB only would probably be more informative, since the WHOQOL-BREF subscales relatively weakly captured the multidimensional character of quality of life in our sample. They produced almost entirely parallel profiles through all four domains which may in fact suggest a univariate character of this questionnaire and it is hard to say whether it stems from sample's specificity or it illustrates more universal pattern.

\section{Conclusions}

Despite these limitations, the study added to the literature on PWB among PLWH by using a person-centred method of analysis that provides insights above and beyond of what can be reached by a variable-centred approach, applied in the majority of previous studies on this topic. More specifically, this approach not only leads to the increase of knowledge on PWB, but may also serve as a base for more effective counselling for PLWH, tailored to the specific needs of different subgroups of patients. Overall, selected personality traits, education and employment turned out to be the key correlates of PWB and outweighed a role of clinical variables, which had no effect on PWB in the studied sample. Thus, it may be tempting to conclude that not the disease itself, but sociodemographic characteristics and personality factors are crucial for PWB among PLWH. However, such conclusion should be treated with caution since our participants can be described as highly functional with effective control of HIV infection and recent findings inform about higher than previously assumed intra- and interindividual variability of PWB in response to demanding circumstances (Diener et al. 2016). 


\section{Compliance with ethical standards}

Disclosure of Potential Conflicts of Interest Author A declares that he has no conflict of interest. Author B declares that she has no conflict of interest. Author $\mathrm{C}$ declares that she has no conflict of interest.

Research Involving Human Participants and/or Animals All procedures performed in studies involving human participants were in accordance with the ethical standards of the institutional and/or national research committee and with the 1964 Helsinki declaration and its later amendments or comparable ethical standards.

Informed Consent Informed consent was obtained from all individual participants included in the study.

Open Access This article is distributed under the terms of the Creative Commons Attribution 4.0 International License (http://creativecommons.org/licenses/by/4.0/), which permits unrestricted use, distribution, and reproduction in any medium, provided you give appropriate credit to the original author(s) and the source, provide a link to the Creative Commons license, and indicate if changes were made.

\section{References}

Alayli, A., Evers, S., \& Alexeeva, D. (2014). A review of economic evaluations of behavior change interventions: Setting an agenda for research methods and practice. Journal of Public Health, 36, 336434. https://doi.org/10.1093/pubmed/fdt080.

Armon, C., \& Lichtenstein, K. (2012). The associations among coping, nadir CD4+ T-cell count, and nonHIV-related variables with health-related quality of life among an ambulatory HIV-positive patient population. Quality of Life Research, 21, 993-1003. https://doi.org/10.1007/s111-0017-2.

Asakawa, K., Senthilselvan, A., Feeny, D., Johnson, J., \& Rolfson, D. (2011). Trajectories of health-related quality of life differ by age among adults: Results from an eight-year longitudinal study. Journal of Health Economics, 31, 207-221. https://doi.org/10.1016/j.jhealeco.2011.

Bogart, L., Wagner, G., Galvan, F., Landrine, H., Klein, D., et al. (2011). Perceived discrimination and mental health symptoms among black men with HIV. Cultural diversity and ethnic minority. Psychology, 17, 295-302. https://doi.org/10.1037/a0024056.

Bullinger, M. (2002). Assessing health related quality of life in medicine. An overview over concepts, methods and applications in international research. Restorative Neurology and Neuroscience, 20, 93101. https://doi.org/10.1457/a0024678.

Burgess, A. P., Carretero, M., Elkington, A., Pasqual-Marsettin, E., Lobacaro, C., \& Catalan, J. (2000). The role of personality, coping style and social support in health related quality of life in HIV infection. Quality of Life Research, 9, 423-437. https://doi.org/10.1023/A:1008918719749.

Cherepanov, D., Palta, M., Fryback, D., Robert, S., Hays, R., et al. (2011). Gender differences in multiple underlying dimensions of health-related quality of life are associated with sociodemographic and socioeconomic status. Medical Care, 49, 1021-1030. https://doi.org/10.1097/MLR.0b013e31822ebed9.

Chi, P., Li, X., Dua, H., Tam, C., Zhao, J., \& Zhao, G. (2016). Does stigmatization wear down resilience? A longitudinal study among children affected by parental HIV. Personality and Individual Differences, 96, 159-163. https://doi.org/10.1016/j.paid.2016.03.001.

Collins, L. \& Lanza, S. (2013). Latent class and latent transition analysis: With applications in the social, behavioral, and health sciences, volume 718. John Wiley \& Sons.

Constanza, R., Fisher, B., \& Ali, S. (2007). Quality of life: An approach integrating opportunities, human needs, and subjective wellbeing. Ecological Economics, 61, 267-276. https://doi.org/10.1016/j. ecolecon.2006.02.023.

Costa Jr., P. T., \& McCrae, R. R. (1992). Revised NEO personality inventory (NEO-PI-R) and NEO five-factor inventory (NEO-FFI) professional manual. Odessa: Psychological Assessment Resources.

Darling, C., Olmstead, S., \& Tigglema, C. (2010). Persons with AIDS and their support persons: Stress and life satisfaction. Stress and Health, 26, 33-44. https://doi.org/10.1002/smi.1254.

Deeks, S., Lewin, S., \& Havlir, D. (2013). The end of AIDS: HIV infection as a chronic disease. Lancet, Published online October, 21, -1533. https://doi.org/10.1016/S0140-6736(13)61809-7.

Degroote, S., Vogelaers, D., \& Vandijck, D. (2014). What determines health-related quality of life among people living with HIV: An updated review of the literature. Archives of Public Health, 72, 40. https://doi. org/10.1186/2049-3258-72-40. 
Diener, E., Emmons, R. A., Larsen, R. J., \& Griffin, S. (1985). The satisfaction with life scale. Journal of Personality Assesment, 49, 71-75. https://doi.org/10.1207/s15327752jpa4901_13.

Diener, E., Oishi, S., \& Ryan, K. (2010). Universals and cultural differences in the causes and structure of happiness: A multilevel review. In C. Keyes (Ed.), Mental well-being (pp. 153-176). Dordrecht: Springer.

Diener, E., Heintzelman, S. J., Kushlev, K., Tay, L., Wirtz, D., Lutes, L. D., \& Oishi, S. (2016). Findings all psychologists should know from the new science on subjective well-being. Canadian Psychology/ Psychologie Canadienne, 58, 87-104. https://doi.org/10.1037/cap0000063.

Erlen, J., Stilley, C., Bender, A., Lewis, M., Garand, L., et al. (2009). Personality traits and chronic illness: A comparison of individuals with psychiatric, coronary heart disease, and HIV/AIDS diagnoses. Applied Nursing Research, 24, 74-81. https://doi.org/10.1016/j.apnr.2009.04.006.

Fassino, S., Leombruni, P., Amianto, F., \& Abbate-Daga, G. (2004). Personality profile of HIV outpatients: Preliminary results and remarks on clinical management. Psychotherapy and Psychosomatics, 73, 361365. https://doi.org/10.1159/000080389.

Fredrickson, B. L. (2013). Updated thinking on positivity ratios. American Psychologist, 68, 814-822. https://doi.org/10.1037/a0033584.

Friedman, H., \& Kern, M. (2013). Personality, well-being, and health. Annual Review of Psychology, 65, 719742. https://doi.org/10.1146/annurev-psych-010213-115123.

Gordillo, V., Fekete, E., Platteau, T., Antoni, M., Schneiderman, N., \& Nöstlinger, C. (2009). Emotional support and gender in people living with HIV: Effects on psychological well-being. Journal of Behavioral Medicine, 32, 53-65. https://doi.org/10.1007/s10865-009-9222-7.

Gravetter, F., \& Wallnau, L. (2014). Essentials of statistics for the behavioral sciences (8th ed.). Belmont: Wadsworth.

Guyatt, G., Feeny, D., \& Patrick, D. (1993). Measuring health-related quality of life. Annals of Internal Medicine, 118, 622-629. https://doi.org/10.1016/0735-1097(93)90488-M.

Jayarajan, N., \& Prabha, S. (2010). HIV and mental health: An overview of research from India. Indian Journal of Psychiatry, 16, 23-39. https://doi.org/10.4103/0019-5545.69245.

Keiser, O., Spycher, B., Rauch, A., Calmy, A., Cavassini, M., Glass, T., Nicca, D., Ledergerber, B., \& Egger, M. (2012). Outcomes of antiretroviral therapy in the Swiss HIV cohort study: Latent class analysis. AIDS and Behavior, 16, 244-255. https://doi.org/10.1007/s10461-011-9971-5.

Liu, C., Ostrow, D., Detels, R., Hu, Z., Johnson, L., Kingsley, L., \& Jacobson, L. (2006). Impacts of HIV infection and HAART use on quality of life. Quality of Life Research, 15, 941-949. https://doi. org/10.1007/s11136-005-5913-X.

Lockenhoff, C., Ironson, G., O’Cleirigh, C., \& Costa, P. (2009). Five-factor model personality traits, spirituality, religiousness, and mental health among people living with HIV. Journal of Personality, 77, 1411-1436. https://doi.org/10.1111/j.1467-6494.2009.00587.x.

Logie, C., \& Gadalla, T. (2009). Meta-analysis of health and demographic correlates of stigma towards people living with HIV. AIDS Care, 21, 742-753. https://doi.org/10.1080/09540120802511877.

Lubke, G. H., \& Neale, M. C. (2006). Distinguishing between latent classes and continuous factors: Resolution by maximum likelihood. Multivariate Behavioral Research, 41(4), 499-532. https://doi. org/10.1207/s15327906mbr4104 4.

McCrae, R., Costa, P., Martin, T., Oryol, V., \& Senin, I. (2007). Personality correlates of HIV stigmatization in Russia and the United States. Journal of Research in Personality, 41, 190-196. https://doi.org/10.1016/j. jrp.2005.11.002.

Moskowitz, J. (2003). Positive affect predicts lower risk of AIDS mortality. Psychosomatic Medicine, 65, 620-626. https://doi.org/10.1097/01.PSY.0000073873.74829.23.

Oberjé, E., Dima, A., van Hulzen, A. W., Prins, J., \& de Bruin, M. (2015). Looking beyond health-related quality of life: Predictors of subjective well-being among people living with HIV in the Netherlands. AIDS and Behavior, 19, 1398-1407. https://doi.org/10.1007/s10461-014-0880-2.

O'Cleirigh, C., Ironson, G., Weiss, A., \& Costa Jr., P. T. (2007). Conscientiousness predicts disease progression (CD4 number and viral load) in people living with HIV. Health Psychology, 26, 473-480. https://doi. org/10.1037/0278-6133.26.4.473.

Odek, W. (2013). Formal employment and health-related quality of life among people living with HIV in South Africa. Applied Research in Quality of Life, 8, 145-168. https://doi.org/10.1007/s11482-012-9183-9.

Pacella, M., Armelie, A., Boarts, J., Wagner, G., Jones, T., Feny, N., \& Delahant, D. (2012). The impact of prolonged exposure on PTSD symptoms and associated psychopathology in people living with HIV: A randomized test of concept. AIDS and Behavior, 16, 1327-1340. https://doi.org/10.1007/s10461-011-0076-y.

Penedo, F. J., Gonzalez, J. S., Dahn, J. R., Antoni, M., Malow, R., Costa, P., \& Schneiderman, N. (2003). Personality, quality of life and HAART adherence among men and women living with HIV/AIDS. Journal of Psychosomatic Research, 54, 271-278. 
Rzeszutek, M., \& Oniszczenko, W. (2013). Association between social support and temperament and the intensity of PTSD symptoms in a sample of HIV-positives. Polish Psychological Bulletin, 44, 431-438. https://doi.org/10.2478/ppb-2013-0046.

Rzeszutek, M., Oniszczenko, W., Żebrowska, M., \& Firlag-Burkacka, E. (2015). HIV infection duration, social support and the level of trauma symptoms in a sample of HIV-positive polish individuals. AIDS Care, 27, 363-369. https://doi.org/10.1080/09540121.2014.963018.

Samji, H., Cescon, A., Hogg, R., Modur, S., Althoff, K., et al. (2013). Closing the gap: Increases in life expectancy among treated HIV-positive individuals in the United States and Canada. PLoS One, 18, 144 156. https://doi.org/10.1371/journal.pone.0081355.

Samson, A., Lavigne, R. M., \& MacPherson, P. (2009). Self-fulfilment despite barriers: Volunteer work of people living with HIV. AIDS Care, 21, 1425-1431. https://doi.org/10.1080/09540120902814403.

Shaheen, A., Ali, U., \& Kumar, H. (2015). Extraversion personality traits and social support as Determinants of coping responses among individuals with HIV/AIDs. Journal of Psychology and Clinical Psychiatry, 4, 1-5. https://doi.org/10.15406/jpcpy.2015.04.00188.

Simmons, Z., Bremer, B., Robbins, R., Walsh, S. M., \& Fischer, B. (2000). Quality of life in ALS depends on factors other than strength and physical function. Neurology, 55, 388-392. https://doi.org/10.1212 /WNL.55.3.388.

Steptoe, A., Deaton, A., \& Stone, A. (2015). Subjective wellbeing, health, and ageing. Lancet, 14, 640-648. https://doi.org/10.1016/S0140-6736(13)61489-0.

Trépanier, L., Rourke, S., Bayoumi, A., Halman, M., Krzyzanowski, S., \& Power, C. (2005). The impact of neuropsychological impairment and depression on health-related quality of life in HIV-infection. Journal of Clinical and Experimental Neuropsychology, 27, 1-15. https://doi.org/10.1080/138033990513546.

Tsevat, J., Leonard, A., Szaflarski, M., Sherman, S., Cotton, S., \& a. (2009). Change in quality of life after being diagnosed with HIV: A multicenter longitudinal study. AIDS Patient Care and STDs, 23, 931-937. https://doi.org/10.1089/apc.2009.0026.

Vassilev, I., Rogers, A., Sanders, C., Cheraghi-Sohi, S., Blickem, C., Brooks, H., \& ... Kennedy, A. (2014). Social status and living with a chronic illness: An exploration of assessment and meaning attributed to work and employment. Chronic Illness, 10, 273-290. https://doi.org/10.1177/1742395314521641.

Vera-Villarroel, P., Valtierra, A., \& Contreras, D. (2016). Affectivity as mediator of the relation between optimism and quality of life in men who have sex with men with HIV. International Journal of Clinical and Health Psychology, 16, 256-265. https://doi.org/10.1016/j.ijchp.2016.07.001.

Vermunt, J. K. (2010). Latent class modelling with covariates: Two improved three-step approaches. Political Analysis, 18, 450-469. https://doi.org/10.1093/pan/mpq025.

Vermunt, J. K., \& Magidson, J. (2004). Latent class analysis. In M. S. Lewis-Beck, A. Bryman, \& T. F. Liao (Eds.), The sage encyclopedia of social sciences research methods (pp. 549-553). Thousand Oaks: Sage Publications.

Ware, J., \& Sherbourne, C. (1992). The MOS 36-item Short-Form Health Survey (SF-36). Conceptual framework and item selection. Medical Care, 30, 473-483. https://doi.org/10.1007/BF03260127.

Watson, D., Clark, L., \& Tellegen, A. (1988). Development and validation of brief measures of positive and negative affect. The PANAS scales. Journal of Personality and Social Psychology, 54, 1063-1070. https://doi.org/10.1053/:102670883.

Whitworth, S., Loftus, A., Skinner, T., Gasson, N., Barker, R., et al. (2013). Personality affects aspects of health-related quality of life in Parkinson's disease via psychological coping strategies. Journal of Parkinson'sDisease, 3, 45-53. https://doi.org/10.3233/JPD-120149.

WHOQOL Group. (1995). WHOQOL Group, The World Health Organization Quality of Life Assessment: position paper from the World Health Organization. Social Science and Medicine, 41, 1403-1409. https://doi.org/10.1016/0277-9536(95)00112-K. 